\title{
Can We Actually Improve Resident Wellness? Pilot Data from a Longitudinal Wellness Curriculum for Emergency Medicine Residents
}

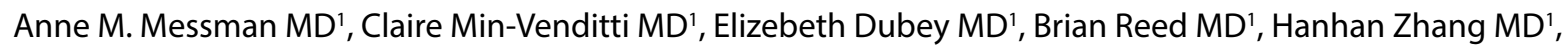 \\ Hafez Bazzi MD², James Paxton MD'
}

\begin{abstract}
Background: The wellness of resident physicians, particularly in emergency medicine (EM) with its high rate of burnout, has become of paramount importance to residency programs. This pilot study aims to evaluate the effects of the implementation of an evidence-based wellness curriculum on self-reported resident wellness across three Detroit EM residency programs. It is the first objective study of a wellness program for EM residents to date.

Methods: This is a prospective, interventional pilot study of the impact of a well-defined, longitudinal wellness curriculum administered to all categorical residents in three EM residency programs in Detroit, Michigan. Anonymous surveys incorporating the Perceived Stress Scale (PSS) and the Connor-Davidson Resilience Scale (CD-RISC) were administered before and after implementation of the wellness curriculum. The curriculum consisted of three modules (Introduction to Wellness, Dealing with Medical Errors, and Shame and Debriefing Traumatic Events in the Emergency Department) over 3 months. Participation was anonymous and voluntary, and all data collection and analysis were provided by a third-party not affiliated with residency program leadership.

Results: A total of 114 residents were enrolled, including 30 (26.3\%) residents who completed both the pre- and post-intervention surveys. These 30 residents demonstrated increased resiliency (i.e., higher mean CD-RISC score), and lower perceived stress (i.e., decreased mean PSS score) following the intervention. However, this difference was only statistically significant with the CD-RISC score $(\mathrm{p}=0.015)$. There was no statistically significant difference on either scoring system with respect to gender, post-graduate year of training, or residency training site.

Conclusions: Implementation of a wellness curriculum in our cohort of residents was shown to improve resilience amongst residents. Gender, post-graduate year of training, and residency training site did not affect these outcomes. This is a milestone accomplishment in the study of wellness in EM residents as no prior intervention has been shown to have a significant positive impact on EM residents.
\end{abstract}

DOI: 10.18297/jwellness/vol1/iss1/7 Received Date: July 26, 2019 Accepted Date: November 20, 2019 Publication Date: December 11, 2019 Website: https://ir.library.louisville.edu/ jwellness/

Recommended Citation: Messman, Anne M.; Min-Venditti, Claire; Dubey, Elizebeth; Reed, Brian; Zhang, Hanhan; Bazzi, Hafez; and Paxton, James (2019) "Can We Actually Improve Resident Wellness? Pilot Data from a Longitudinal Wellness Curriculum for Emergency Medicine Residents," Journal of Wellness: Vol. 1 : Iss. 1 , Article 7.

Affiliations:

'Wayne State University School of

Medicine

${ }^{2}$ St. John Hospital and Medical Center

\section{INTRODUCTION}

Resident physician wellness is a topic of increasing interest in the medical community. The importance of formalized avenues to support physician well-being is becoming more widely acknowledged, and open discourse regarding the mental and physical toll of physicians' stressful and demanding profession is taking place more readily than in the past.

Burnout, defined as a syndrome of emotional exhaustion, depersonalization and feelings of inadequacy, is strikingly common in medicine, occurring at much higher rates among physicians than within the general population [1-3]. Burnout yields wide and serious consequences. It has been linked to increased unprofessional behavior, increased medical errors, earlier career termination, reduced patient satisfaction, reduced empathy, and high rates of depression and suicide $[4,5]$. Physicians in training appear to be particularly susceptible [6,7]. One large study found the self-reported rate of burnout among residents to be $51.5 \%$ [8]. In fact, residency training has been and remains a period in which physicians lack sufficient sleep and exercise, may not eat well, and are apt

${ }^{*}$ Correspondence To: Anne M. Messman MD

Email: amessman@med.wayne.edu to experience difficult family interactions [9]. Burnout seems to be even worse in specialties on the front lines of care, including emergency medicine [2]. A recent survey of emergency medicine residents reported a burnout rate of 76.1\% amongst the respondents [10]. For the sake of both physicians and patients, we must address and mitigate burnout in medicine.

Residency training is a crucial time in laying the foundation for practice patterns that promote physician wellness. Physicians and medical educators must find a way to incorporate wellness into resident education so that it commands legitimate respect as part of educating good doctors [11]. The Accreditation Council for Graduate Medical Education (ACGME) recently outlined the expectation that residency programs incorporate education on burnout prevention, further substantiating the importance of making this an established part of physician education [12]. A recent multi-center survey determined that emergency medicine residents considered wellness to be valuable to their learning and believed it to be an important topic to include in training, but they did not feel well 
versed in wellness principles [13]. In other words, there is both a desire amongst physicians in training to be educated on wellness, as well as an unmet need.

Several institutions have explored formalized efforts to increase wellness education in physician training. A 2016 study demonstrated the efficacy of a wellness and suicide prevention program for residents that included counseling, psychiatric evaluations and wellness workshops. This intervention was met with high utilization of services and high levels of satisfaction, highlighting the practical and valuable nature of formalized wellness programs [12]. Another study showed that after implementation of a wellness program focused on healthy coping mechanisms and diminishing stressors, anesthesia residents demonstrated improved problem solving, felt more social support in the workplace, and reported less stress and anxiety [13]. Several systematic reviews have yielded similarly positive results, one showing that interventions targeting health and coping mechanisms seem to improve resident well-being, and another finding that $80 \%$ of burnout intervention programs were successful in reducing burnout $[14,15]$.

The aim of this study is to examine the effect of implementation of an evidence-based, longitudinal curriculum for residency physician wellness on selfreported resident wellness at three Detroit emergency medicine residency programs [16]. This curriculum was designed explicitly for EM residents with the support of the Wellness Think Tank, a wellness initiative sponsored by Academic Life in EM (ALiEM). While previous studies have made broad recommendations to improve emergency medicine resident wellness at the level of the institution and the individual, this is one of the first studies to evaluate this particular curriculum to date [17]. We hypothesize that residents would report decreased perceived stress and increased resiliency, measured using two well-validated surveys, after completion of three months of the wellness curriculum. This study will help contribute to the growing literature on techniques and interventions to improve resident physician wellness. It will also serve as a first step to validate the proposed wellness curriculum, which may more readily allow promotion and dissemination of a potentially useful and generalizable method for promoting wellness in residency programs.

\section{METHODS}

\section{Study design}

This was a prospective, interventional study of an intervention (wellness curriculum) among categorical residents in emergency medicine training. This study was approved by the Wayne State University (WSU) Institutional Review Board (IRB). No funding was provided from any source for this study.

\section{Study Setting and Population}

The population of interest included resident physicians in training at three distinct emergency medicine residencies, all affiliated with Wayne State University, Detroit, Michigan. All three residency programs are 3 -year categorical programs. Resident physicians from all 3 years of residency training were included in the study population. No residents were excluded from participation in the study. A total of 114 categorical residents were eligible for participation across all 3 sites. Completion of the wellness curriculum itself was mandatory for all residents. The intervention consisted of resident wellness sessions held during weekly departmental grand rounds, and attendance was required for all resident physicians. However, participation in the pre- and post-intervention surveys was both voluntary and anonymous.

\section{Study Protocol}

In February 2018, prior to the initiation of the threemonth wellness curriculum pilot, a SurveyMonkey (www.surveymonkey.com) survey was sent to all residents, to ascertain which two wellness modules were of most interest to participating residents from all of the available modules in the curriculum. The intention was that the "Introduction to Wellness" module would be provided by study personnel to all residents in March 2018, and the two top-ranked wellness modules, as voted on by the residents via the SurveyMonkey survey, would be provided in April and May 2018. Ultimately, the two most popular wellness modules were "Dealing with Medical Errors," and "Shame and Debriefing Traumatic Events in the Emergency Department." These were presented in April and May 2018, respectively. These two modules were overseen by program leadership at each site, based on the wellness curriculum provided in the Wellness Think Tank.

Identical pre- and post-intervention Qualtrics ${ }^{\circledast}$ surveys were collected from residents during a window from 21 February 2018 to 8 March 2018 for the preintervention survey, and 9 June 2018 to 25 June 2018 for the post-intervention survey. All survey responses were anonymous, and completion of the surveys was described to the residents as voluntary. The pre- and post-intervention surveys collected non-identifiable demographic information from all participating residents, including residency site, gender and postgraduate year (PGY). Each respondent to the Qualtrics ${ }^{\circledast}$ survey self-assigned a unique identifier for use on both the pre- and post-intervention surveys (i.e., "name of street that the respondent grew up on" + "year of high school graduation," ex. Westchester2000). In addition to demographic data, the pre- and post-intervention surveys incorporated both the Perceived Stress Scale (PSS) and the Connor-Davidson Resilience Scale (CD-RISC). The PSS is a widely-used psychological instrument to measure the user's perception of stress, and CD-RISC is a 25-item measure of resilience that has previously been shown to be both valid and reliable $[18,19]$. All data from the pre- and post-intervention surveys were collected and analyzed by a third-party member of the WSU faculty (JHP), who is not affiliated with the residency program leadership. Survey data were matched and de-identified by the third-party faculty member prior to distribution to the remainder of the study team.

\section{Measurements}

The complete text of the PSS is openly available online, free of charge however the CD-RISC scale charges a nominal fee for usage and therefore we cannot provide those survey questions [20]. Identical questions, incorporating both the PSS and CDRISC questionnaires, were included in both surveys. Responses were downloaded into Microsoft ${ }^{\oplus}$ Excel files, and matched by unique identifiers to link pre- and postintervention survey responses.

The PSS consists of 10 questions (i.e., survey questions \#5-14), which are scored by reversing responses (e.g., $0=4,1=3$; etc) to the four positively-stated items (i.e., survey questions $\# 8,9,11,12)$, and then summing across 
all scale items. The full range of scores is from $0-40$ points, with higher scores reflecting a greater degree of perceived stress.

The CD-RISC consists of 25 questions (i.e., survey questions \#15-39), which are scored by summing the total of all items, each of which is scored from 0-4 points. Therefore, the full range of scores is from 0-100 points, with higher scores reflecting greater resilience.

\section{Data Analysis}

Data from the pre- and post-intervention surveys were matched by unique identifier, and comparison was made across pre- and post-test surveys by the third-party faculty member. Total summed scores were computed by the third-party faculty member and de-identified prior to analysis by the WSU institutional biostatistical service. Data were provided to the WSU biostatistical service in Microsoft ${ }^{\otimes}$ Excel format, and statistical analysis was conducted by the WSU biostatistical service, using Statistical Analysis Software (SAS), version 9.4 (SAS

Table 1: Score Comparisons by Gender for All Subjects

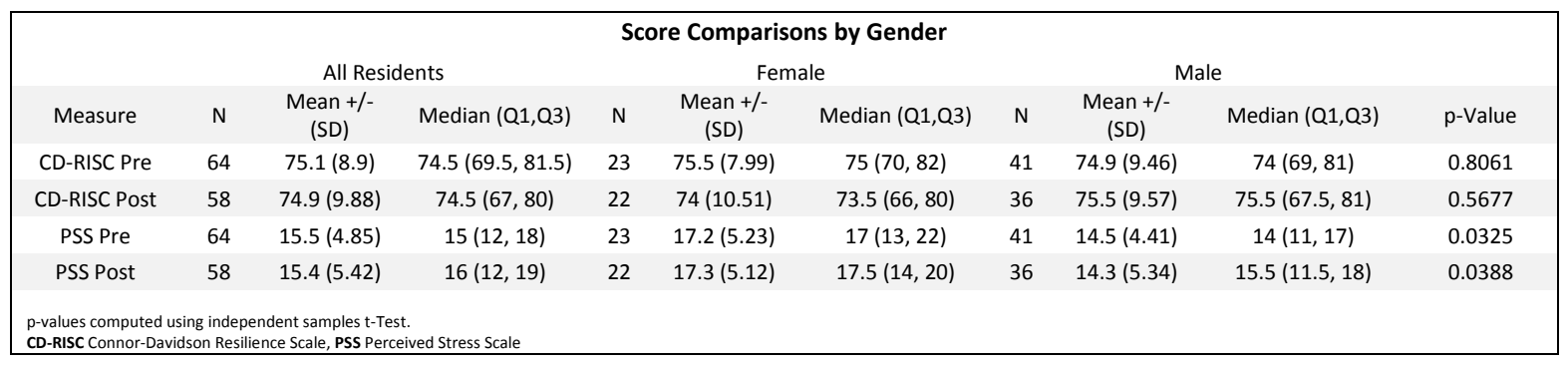

Institute, Cary NC). Comparison of pre- and postintervention survey results was performed, reporting mean (with standard deviation) and median (with interquartile ranges) values, with p-values according to standard techniques. Subgroup analysis was done according to residency program (St. John Hospital [SJH], Sinai-Grace Hospital [SGH], Detroit Receiving Hospital [DRH]), gender, and post-graduate year. Scores for all instrument measures were assessed for normality using both the Shapiro-Wilk and Kolmogorov-Smirnov tests. No significant departures from normality were observed on either instrument for either time period. The p-values for score comparison by gender were computed using independent sample t-tests. Comparison of preand post-intervention scores for residents completing both evaluations were performed with dependent sample $\mathrm{t}$-tests.. The $\mathrm{p}$-values for categorical comparison by gender were computed using Chi-Square test.
The p-values for categorical comparisons of resident by year of training and training site were computed using Chi-Square test for gender and year, and Fisher's Exact test for PSS and CD-RISC improvement scores. The p-values computed for score comparisons by site were conducted using one-way ANOVA.

\section{RESULTS}

A total of 64 of 114 residents $(56.1 \%)$ completed the combined CD-RISC / PSS pre-intervention survey, and a total of 58 residents $(50.9 \%)$ completed the combined post-intervention survey (Table 1). The mean preintervention PSS score for all participants was 15.5 (SD $+/-4.85$ ), and the mean post-intervention PSS score was 15.4 (SD +/- 5.42) points. The mean pre-intervention CD-RISC score for all participants was 75.1 (SD +/$8.9)$, and the mean post-intervention CD-RISC score was 74.9 (SD +/- 9.88). Score comparison by gender demonstrated a statistically significant difference with both pre- and post-intervention PSS surveys, but not with either of CD-RISC surveys, when all subjects were included (Table 1).
A total of 30 of 114 (26.3\%) residents completed both the pre- and post-intervention surveys. The mean "delta" (post-intervention score minus pre-intervention score) is provided for both the CD-RISC and PSS surveys in Table 2. These 30 residents demonstrated increased resiliency (i.e., higher mean CD-RISC score), and lower perceived stress (i.e., decreased mean PSS score) following the intervention. However, this difference was only statistically significant with the CD-RISC score $(\mathrm{p}=0.015)$. Neither CD-RISC nor PSS showed a statistically significant difference with respect to subject gender (Table 2), even when subjects were evaluated by site and training year. None of the scores differed significantly with respect to resident post-graduate year (Table 3), and no variables showed a significant difference with respect to resident post-graduate year (Table 4-5). Pre-intervention PSS scores were significantly different across residency training sites, but none of the categorical variables showed significant differences with respect to residency training site (Table 6). See Appendix A for tables 3-6.

Table 2: Score Comparisons by Gender for Those 30 Subjects Who Completed Both Surveys

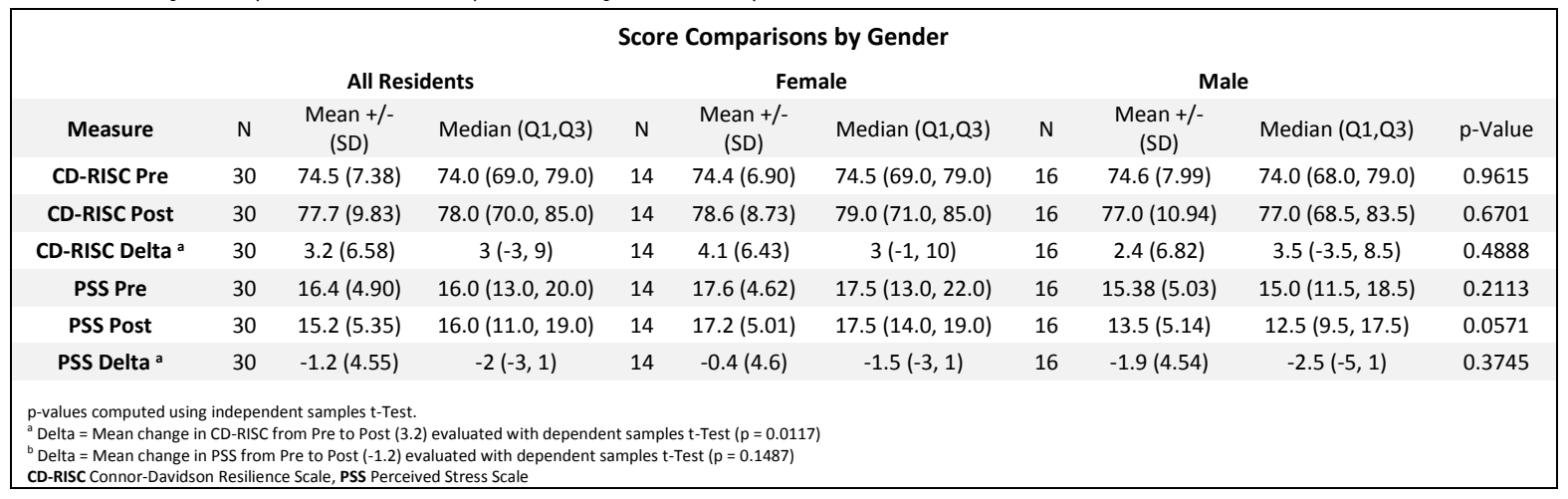




\section{DISCUSSION}

Previous research has determined that resident physicians enter residency with a higher-than-average prevalence of distress [21]. This highlights the importance of enacting a wellness curriculum that actually works, given that resident physicians are already starting their training at a disadvantage. We sought to evaluate the efficacy of one such wellness curriculum and although only three of the available 17 modules were presented in this pilot curriculum, the fact that resilience increased in such a short time frame is encouraging.

The wellness curriculum piloted in this study was exclusively designed by and for emergency medicine residents and was created over a one-year time period by the Wellness Think Tank, a working group overseen by Academic Life in Emergency Medicine (ALiEM) [16]. The Wellness Think Tank is comprised of resident members from all over the United States, with oversight by faculty members at various institutions nationally. From 2016-2017, the Wellness Think Tank met primarily asynchronously, and once in person at the first-ever Resident Wellness Consensus Summit in May 2017, to establish the curriculum. The end-result was a 17-module wellness curriculum that was made open-access in February 2018 as an appendix to the article describing the process by which the curriculum was developed. It is the first comprehensive wellness curriculum to be published specifically for emergency medicine residents. This study sought to pilot this curriculum to evaluate its effectiveness in decreasing perceived stress and increasing resilience amongst emergency medicine residents at three residency programs in Detroit.

"Wellness" and "physician well-being" are themselves difficult to define and objectively measure, therefore we used multiple objective surrogates to measure "wellness"in our resident cohort. Rather than creating a novel, unvalidated survey instrument for this study, the PSS and CD-RISC were chosen specifically since they had already undergone psychometric testing and validation. Of all available wellness assessment tools, these two were chosen specifically based on expert recommendation from senior leadership from the Wellness Think Tank. An additional benefit was that they were both low- or nocost to administer. Perceived stress has been previously shown to increase the chance of suicidal ideation in PGY-1 residents and to be a predictor of post-graduate mental health problems; it is for these reasons that the PSS was chosen to be included in the survey instrument $[22,23]$. Physician resilience has been shown to increase well-being and is protective of burnout and therefore the CD-RISC was also included in our survey instrument [24]. In addition to the PSS and CD-RISC, the survey instrument also acquired demographic data. We were careful to collect demographic data that was interesting and relevant (institution where the resident is training, gender and PGY level of training) but ensured that data was not collected that would potentially void the anonymity of the respondent. For this reason, further demographic information, such as age, was not collected.

Although we were unable to demonstrate a significant improvement in perceived stress during the piloting of the wellness curriculum (Table 2 mean decrease in perceived stress $-1.2, \mathrm{p}=0.1487$ ), our research did show a statistically significant improvement in resilience (Table 2, mean increase in resilience of 3.2, $\mathrm{p}=0.0117$ ) amongst those that responded to both the pre- and post-intervention surveys. This is a milestone accomplishment in the study of wellness in emergency medicine residents as no prior intervention has been shown to have a significant positive impact on emergency medicine residents. In fact, this study is one of the first residency wellness curriculums to be studied among emergency medicine residents. A recently-published systematic review of interventions to reduce resident physician burnout identified only 19 articles, out of a pool of 1870 , that met the author's inclusion criteria, which included studies that presented original data, enrolled residents, had an identifiable intervention with followup results, and were published in English-language, peer-reviewed medical journals [25]. None of the 19 studies were in the field of emergency medicine. Our study is the first of its kind and comes at an incredibly important time, given the high rates of burnout amongst emergency physicians that seem to be worsening every year [26]. Additionally, our study was multi-institutional, thus increasing our sample size and making our results more generalizable. Small sample size and research being conducted within a single residency program have been noted previously to diminish the generalizability of the few studies on interventions to increase wellness and decrease burnout [25].

It is important to note that our results do not suggest a statistically significant difference in any of the subgroup analyses of the data. There was no difference in responses as distinguished by gender, site of training or PGY level. This implies that the curriculum may benefit all residents, given that no group seemed to be "more well" than any other group.

\section{LIMITATIONS}

Although the results of this study are encouraging, there are significant limitations which can be addressed in the future. As this was a pilot curriculum, only three months of material were covered. Although care was taken to identify the modules most important to the resident physicians, findings of greater significance will be identified when the curriculum is studied over a longer period of time and with more modules presented. Another significant limitation was the response rate to the surveys pre- and post-intervention. Although the pre- and post-intervention survey response rates were $56.1 \%$ and $50.9 \%$, respectively, only $26.3 \%$ responded to both the pre- and post-intervention survey, making it difficult to establish generalizable conclusions. An increased response rate would further elucidate the effectiveness of the wellness curriculum. Although we performed subgroup analyses on post-graduate year, gender, and residency site, this study is not powered for such an analysis. Furthermore, since this was a pilot study, we had no previous studies to help us predict what a clinically-significant "delta" for improvement in either metric should be. Lastly, although we did show improvement in resilience based on a psychometric tool to measure resilience, we did not measure what tangible outcomes this may have had on the residents.

Additionally, the pre-intervention survey was given in February and the post-intervention survey was given in June, so seasonal variations could affect the responses provided on the surveys, potentially inflating the June scores. Conversely, advancement to the next post-graduate year and its concomitant increase in responsibility, including graduation from residency, may adversely affect survey responses. We believe that longerterm studies will help to mitigate this effect. Although our study was multi-institutional, it was limited to residency programs in the city of Detroit, which may limit the generalizability of the results outside of this geographic area. 
Finally, the wellness curriculum was presented during weekly didactics. Although attendance to $75-80 \%$ of didactic sessions is mandatory, some are excused from didactics because of clinical responsibilities and others are absent from didactics for personal reasons. It is unknown whether the survey respondents attended any or all of the wellness sessions, and this may positively or negatively impact the survey results. Attendance of the residents at these sessions can be tracked but not definitively paired with the respondents because of the anonymous nature of the survey. Future studies may attempt to correlate resident attendance with survey responses however care will need to be exercised in order to retain the anonymity of the respondents.

\section{CONCLUSIONS}

Resident physician wellness is an important topic, particularly in emergency medicine, which is frequently cited as having one of the highest burnout rates of all fields of medicine [2]. As with any other curricular intervention, wellness curricula need to be evaluated to establish their efficacy and modified as necessary. We present the piloting of a comprehensive, resident-created wellness curriculum shown to improve resilience over a three-month time period. Although future studies are necessary to determine long-term effects of the curriculum and in more diverse resident cohorts, our work represents one of the first wellness curriculums for resident physicians within emergency medicine. This is a significant step forward in a movement to improve the wellness of resident physicians and decrease their burnout, and will prepare resident physicians for a long, successful career within emergency medicine.

\section{REFERENCES}

1. Lee RT, Ashforth BE. On the meaning of Maslach's three dimensions of burnout. J Appl Psychol. 1990 Dec;75(6):743-7.

2. Shanafelt TD, Boone S, Tan L, Dyrbye LN, Sotile W, Satele D, et al. Burnout and satisfaction with work-life balance among US physicians relative to the general US population. Arch Intern Med. 2012 Oct;172(18):1377-85.

3. Lindeman S, Laara E, Hakko H, Lonnqvist J. A systematic review on gender-specific suicide mortality in medical doctors. Br J Psychiatry. 1996 Mar;168(3):274-9.

4. Casalino LC, Crosson FJ. Physician Satisfaction and Physician Well-Being: Should Anyone Care? Professions and Professionalism. 2015;5(1):954.

5. Blackwelder R, Watson KH, Freedy JR. Physician Wellness Across the Professional Spectrum. Prim Care. 2016 Jun;43(2):355-61.

6. Hull SK, DiLalla LF, Dorsey JK. Prevalence of health-related behaviors among physicians and medical trainees. Academic psychiatry : the journal of the American Association of Directors of Psychiatric Residency Training and the Association for Academic Psychiatry 2008;32:31-8. https://doi. org/10.1176/appi.ap.32.1.31.

7. Sargent MC, Sotile W, Sotile MO, Rubash H, Barrack RL. Stress and coping among orthopaedic surgery residents and faculty. The Journal of bone and joint surgery American volume 2004;86-a:1579-86. https://doi.org/10.2106/00004623-200407000-00032.

8. West CP, Shanafelt TD, Kolars JC. Quality of life, burnout, educational debt, and medical knowledge among internal medicine residents. JAMA. 2011 Sep;306(9):952-60.

9. Perry MY, Osborne WE. Health and wellness in res idents who matriculate into physician training programs. Am J Obstet Gynecol. 2003 Sep;189(3):679-83.

10. Lin M, Battaglioli N, Melamed M, Mott SE, Chung AS, Robinson DW. High Prevalence of Burnout Among US Emergency Medicine Residents: Results From the 2017 National Emergency Medicine Wellness Survey. Ann Emerg Med. 2019 Nov;74(5):682-90.

11. El-Shafei DA, Abdelsalam AE, Hammam RA, Elgohary $\mathrm{H}$. Professional quality of life, wellness education, and coping strategies among emergency physicians. Environ Sci Pollut Res Int. 2018 Mar;25(9):9040-50.

12. Ey S, Moffit M, Kinzie JM, Brunett PH. Feasibility of a Comprehensive Wellness and Suicide Prevention Program: A Decade of Caring for Physicians in Training and Practice. J Grad Med Educ. 2016 Dec;8(5):747-53.

13. Saadat H, Snow DL, Ottenheimer S, Dai F, Kain ZN. Wellness program for anesthesiology residents: a randomized, controlled trial. Acta Anaesthesiol Scand. 2012 Oct;56(9):1130-8.

14. Raj KS. Well-Being in Residency: A Systematic Review. J Grad Med Educ. 2016 Dec;8(5):674-84.

15. Awa WL, Plaumann M, Walter U. Burnout prevention: a review of intervention programs. Patient Educ Couns. 2010 Feb;78(2):184-90.

16. Arnold J, Tango J, Walker I, Waranch C, McKamie J, Poonja Z, et al. An Evidence-based, Longitudinal Curriculum for Resident Physician Wellness: The 2017 Resident Wellness Consensus Summit. West J Emerg Med. 2018 Mar;19(2):337-41.

17. Ross S, Liu EL, Rose C, Chou A, Battaglioli N. Strategies to Enhance Wellness in Emergency Medicine Residency Training Programs. Ann Emerg Med. 2017 Dec;70(6):891-7.

18. Connor KM, Davidson JR. Development of a new resilience scale:the Connor-Davidson Resilience Scale (CD-RISC). Depress Anxiety. 2003;18(2):76-82.

19. Taylor JM. Psychometric analysis of the TenItem Perceived Stress Scale. Psychol Assess. 2015 Mar;27(1):90-101.

20. Ishak WW, Lederer S, Mandili C, Nikravesh R, Seligman L, Vasa M, et al. Burnout during residency training: a literature review. J Grad Med Educ. 2009 Dec;1(2):236-42.

21. Dyrbye LN, Moutier C, Durning SJ, Massie FS Jr, Power DV, Eacker A, et al. The problems program directors inherit: medical student distress at the time of graduation. Med Teach. 2011;33(9):756-8.

22. Tyssen R, Vaglum P, Grønvold NT, Ekeberg O. Factors in medical school that predict postgraduate mental health problems in need of treatment. A nationwide and longitudinal study. Med Educ. 2001 Feb;35(2):110-20.

23. Tyssen R, Vaglum P, Grønvold NT, Ekeberg O. Suicidal ideation among medical students and young physicians: a nationwide and prospective study of prevalence and predictors. J Affect Disord. 2001 Apr;64(1):69-79.

24. Epstein RM, Krasner MS. Physician resilience: what it means, why it matters, and how to promote it. Acad Med. 2013 Mar;88(3):301-3.

25. Busireddy KR, Miller JA, Ellison K, Ren V, Qayyum R, Panda M. Efficacy of Interventions to Reduce Resident Physician Burnout: A Systematic Review. J Grad Med Educ. 2017 Jun;9(3):294-301.

26. Shanafelt TD, Hasan O, Dyrbye LN, Sinsky C, Satele $\mathrm{D}$, Sloan J, et al. Changes in Burnout and Satisfaction With Work-Life Balance in Physicians and the General US Working Population Between 2011 and 2014. Mayo Clin Proc. 2015 Dec;90(12):1600-13. 
Appendix A: Tables 3-6

Table 3: Score Comparisons by Postgraduate Year for All Subjects

\begin{tabular}{|c|c|c|c|c|c|c|c|c|c|c|}
\hline \multicolumn{11}{|c|}{ Score Comparisons by Postgraduate Year } \\
\hline \multicolumn{5}{|c|}{ Year 1} & \multicolumn{2}{|c|}{ Year 2} & \multicolumn{3}{|c|}{ Year 3} & \multirow[b]{2}{*}{$p$-Value } \\
\hline Measure & $\mathrm{N}$ & Mean (SD) & Median (Q1, Q3) & $\mathrm{N}$ & Mean (SD) & Median (Q1, Q3) & $\mathrm{N}$ & Mean (SD) & Median (Q1, Q3) & \\
\hline Pre CD-RISC & 26 & $74.8(8.55)$ & $75.5(70,81)$ & 23 & $73.9(9.06)$ & $73(69,81)$ & 15 & $77.5(9.39)$ & $79(71,83)$ & 0.4640 \\
\hline Post CD-RISC & 23 & $75.8(10.15)$ & $76(66,85)$ & 20 & 75 (10.53) & $76(68,84)$ & 15 & $73.5(9)$ & $74(66,77)$ & 0.7842 \\
\hline Pre PSS & 26 & $16.5(4.47)$ & $17(14,19)$ & 23 & $14.8(5.66)$ & $13(10,17)$ & 15 & $14.6(4.03)$ & $14(13,18)$ & 0.3377 \\
\hline Post PSS & 23 & $16.6(3.37)$ & $17(13,19)$ & 20 & $14.5(6.89)$ & $16(9,19)$ & 15 & $14.9(5.79)$ & $13(11,17)$ & 0.4134 \\
\hline
\end{tabular}

\begin{tabular}{|c|c|c|c|c|c|c|}
\hline \multicolumn{7}{|c|}{ Categorical Comparisons by Postgraduate Year } \\
\hline Variable & Value & All & First Year & Second Year & Third Year & p-Value \\
\hline \multirow[t]{2}{*}{ Gender } & Female & $31(33.7)$ & $10(28.6)$ & $16(48.5)$ & $5(20.8)$ & 0.0666 \\
\hline & Male & $61(66.3)$ & $25(71.4)$ & $17(51.5)$ & $19(79.2)$ & \\
\hline \multirow[t]{2}{*}{ PSS } & No Improvement & $12(40)$ & $6(42.9)$ & $4(40)$ & $2(33.3)$ & 1 \\
\hline & Score Improved & $18(60)$ & $8(57.1)$ & $6(60)$ & $4(66.7)$ & \\
\hline \multirow[t]{2}{*}{ CD-RISC } & No Improvement & $12(40)$ & $5(35.7)$ & $4(40)$ & $3(50)$ & 0.89063 \\
\hline & Score Improved & $18(60)$ & $9(64.3)$ & $6(60)$ & $3(50)$ & \\
\hline \multirow[t]{3}{*}{ Site } & DRH & $27(29.3)$ & $11(31.4)$ & $11(33.3)$ & $5(20.8)$ & 0.4855 \\
\hline & SGH & $38(41.3)$ & $16(45.7)$ & $10(30.3)$ & $12(50)$ & \\
\hline & $\mathrm{SJH}$ & $27(29.3)$ & 8 (22.9) & $12(36.4)$ & $7(29.2)$ & \\
\hline
\end{tabular}

Table 5: Comparison of Change in Survey Scores By Year for Those 30 Subjects Who Completed Both Surveys

\begin{tabular}{|c|c|c|c|c|c|c|c|c|c|c|}
\hline \multicolumn{11}{|c|}{ Comparison of Change in Survey Scores by Year } \\
\hline \multicolumn{5}{|c|}{$1^{\text {st }}$ Year } & \multicolumn{2}{|c|}{$2^{\text {nd }}$ Year } & \multicolumn{3}{|c|}{$3^{\text {rd }}$ Year } & \multirow[b]{2}{*}{$\mathrm{p}$-Value } \\
\hline Measure & $\mathrm{N}$ & Mean (SD) & Median (Q1, Q3) & $\mathrm{N}$ & Mean (SD) & Median (Q1, Q3) & $\mathrm{N}$ & Mean (SD) & Median (Q1, Q3) & \\
\hline $\begin{array}{l}\text { CD-RISC } \\
\text { Post - Pre }\end{array}$ & 14 & $2.9(7.23)$ & $3(-4,9)$ & 10 & $5(6.55)$ & $5(-1,11)$ & 6 & $1(5.1)$ & $0.5(-3,5)$ & 0.5028 \\
\hline $\begin{array}{l}\text { PSS Post - } \\
\text { Pre }\end{array}$ & 14 & $-1(4.45)$ & $-1(-3,1)$ & 10 & $-2.3(4.24)$ & $-2.5(-6,1)$ & 6 & $0(5.66)$ & $-2.5(-3,1)$ & 0.6150 \\
\hline
\end{tabular}

Table 6: Comparison of Change in Survey Scores By Site for Those 30 Subjects Who Completed Both Surveys

\begin{tabular}{|c|c|c|c|c|c|c|c|c|c|c|}
\hline \multicolumn{11}{|c|}{ Comparison of Change in Survey Scores by Site } \\
\hline \multirow[b]{2}{*}{ Measure } & \multicolumn{3}{|c|}{ SGH } & \multicolumn{3}{|c|}{ DRH } & \multicolumn{3}{|c|}{ SJH } & \multirow[b]{2}{*}{ p-Value } \\
\hline & $\mathrm{N}$ & Mean (SD) & Median (Q1, Q3) & $\mathrm{N}$ & Mean (SD) & Median (Q1, Q3) & $\mathrm{N}$ & Mean (SD) & Median (Q1, Q3) & \\
\hline $\begin{array}{c}\text { CD-RISC } \\
\text { Post - Pre }\end{array}$ & 13 & $4.1(7.2)$ & $3(0,9)$ & 10 & $1.3(6.5)$ & $-1.5(-3,7)$ & 7 & $4.4(5.9)$ & $5(-2,8)$ & 0.5368 \\
\hline $\begin{array}{l}\text { PSS Post - } \\
\text { Pre }\end{array}$ & 13 & $-0.7(5.3)$ & $1(-3,3)$ & 10 & $-1.5(5.1)$ & $-2(-4,0)$ & 7 & $-1.9(2)$ & $-3(-3,1)$ & 0.8491 \\
\hline
\end{tabular}

